\title{
A new surgical method of pilonidal sinus treatment: A bilaterally paralel elliptic fascio-cutaneous advancement flap technique
}

\author{
Yuksel BC, Berkem H, Ozel H, Hengirmen S \\ Department of General Surgery, Ankara Numune Education and Research Hospital, Sihhiye, Ankara, Turkey. \\ bulentcyuksel@yahoo.com.tr
}

\begin{abstract}
Objective: Pilonidal sinus disease (PSD) is a common chronic disease in young people, It is often associated with considerable discomfort and morbidity

Background: We aimed to describe our new bilateral parallel elliptic fascio-cutaneous advancement flap technique in PSD treatment and we assessed the technique's results in 57 patients.

Method: This procedure consists of a bilateral parallel elliptical excision, mobilization of the fascio-cutaneous flap from the median line of the wound, fixation of the base of the flap to the sacrococcygeal fascia by method of overlapping, and suturing its edge to the lateral side. Finally, we closed the wound without tension in accord with the anatomical plane.

Result: The mean age of the patients was $25.3 \pm 4$ years. Complications such as infection, wound dehiscence, and seroma were detected in $2(3.5 \%), 2(3.5 \%)$, and $3(5.2 \%)$ patients, respectively. The mean durations of hospitalization and absence from work were $2.4 \pm 3$ and $12.6 \pm 3$ days, respectively. The mean length of defects after operations was $14.4 \pm 1 \mathrm{~cm}$ and the mean width was $7.2 \pm 1 \mathrm{~cm}$ ). Follow up period averaged $21.4 \pm 1.2$ (range, 12 to 72 ) months. Recurrence occurred in one (1.7\%) patient included in this study. We detected a mild-degree wound dehiscence in patients with wound infection $(n=2,3.5 \%)$.

Conclusion: Our novel technique provided the patients with minimum postoperative morbidity, short hospital stay and reduced absence from work. In addition, the technique has a satisfying aesthetic outcome and a decreased recurrence rate. Moreover, the flap could be prepared easily. In the light of our results, we suggest that our novel surgical technique seems to be a reasonable method in treatment of PSD (Fig. 2, Ref. 24). Full Text in PDF www.elis.sk.

Key words: fascio-cutaneous advancement flap, pilonidal sinus disease, surgical treatment.
\end{abstract}

Pilonidal Sinus Diseases (PSD) is a common chronic disease in young people. It is often associated with considerable discomfort and morbidity. Although various surgical techniques are currently used for treatment of PSD, no clear consensus as to optimal treatment has been reported so far in the literature $(1,2)$. Apart from the controversy over the best surgical technique for PSD treatment, an ideal operation should be simple, not require a prolonged hospital stay, have a low recurrence rate, be associated with minimal pain and wound care, decrease the period of absence from work $(2,3)$, and consequently lead to patient's aesthetic satisfaction (4, 5). Several studies reported that lateralization of the natal cleft is important in prevention of midline recurrences $(2,6-8)$. Oblique or asymmetric closure techniques such as Karydakis flap and the Bascon procedure are based on this principle $(6,7)$. Using the excision and closure with transposition or advancement flap has received growing attention in recent years because of low recurrence rates demonstrated with those techniques $(4,8-10)$.

Department of General Surgery, Ankara Numune Education and Research Hospital, Sihhiye, Ankara, Turkey.

Address for correspondence: B.C. Yuksel, MD, Ankara Numune Education and Research Hospital, General Surgery, Sihhiye, 06680, Ankara, Turkey.

Phone: +90.0.312.2807038, Fax: +90.0.312.4182760
Taking advantage of advancement flap design with greater flexibility and safe coverage, the transposition and advancement flap have become common techniques for PSD treatment. Nevertheless, the risk of dehiscence, distal necrosis and aesthetic dissatisfaction are important problems in these techniques $(5,8-10)$. On the basis of this issue, we developed a new technique for dealing with afore-mentioned problems. By using this novel technique, we have successfully treated a PSD patient with an appropriate flap design for excised natal cleft and thus avoided the patient's consequent aesthetic dissatisfaction.

In the present study, we aimed to describe our new bilaterally parallel elliptical fascio-cutaneous advancement flap technique in PSD treatment, and we assessed this technique's results in 57 patients.

\section{Method}

This prospective cohort study was carried out in 57 consecutive patients treated with PSD. From January 2005 to February 2008, 57 patients (48 male, 9 female) with PSD were reconstructed with our new technique. Exclusion criteria included age below 15 years, collagen tissue disease, and bilaterally generalized sinus pore out of the navicular area (11).

The data obtained and noted included gender, age, type of disease (primary or recurrent), duration of hospital stay, and compli- 

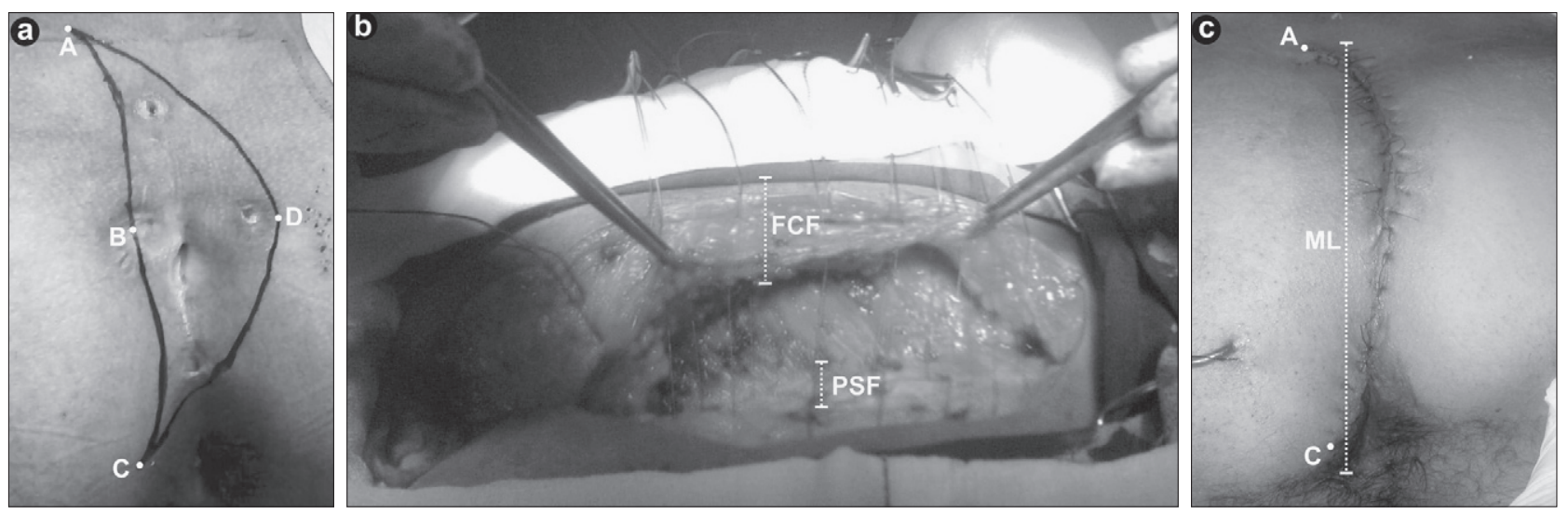

Fig. 1. Flap mapping onto the skin (a), the D-flap starts by excising all sinuses down to the presacral fascia using an asymmetric elliptical incision and creating a new fascio-cutaneous flap; (b) postoperative appearance of the D-flap (c). (FCF - fascio-cutaneous flap, PSF - presakral fascia, ML - mid-line)
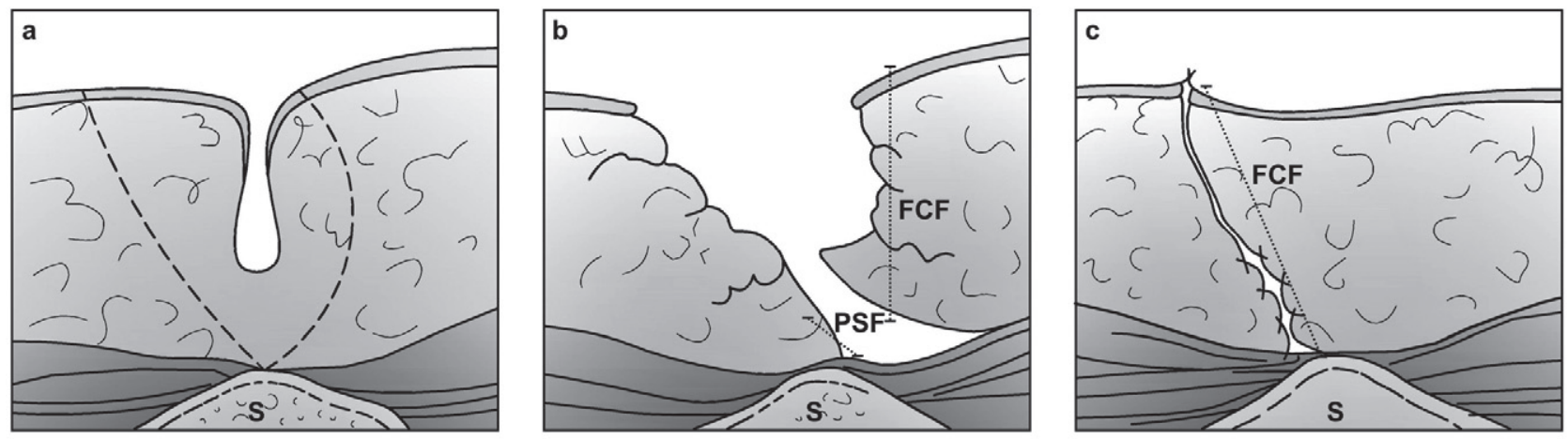

Fig. 2. Excision of the disease and primary closure is one of new fascio-cutaneous advancement flap (D-flap) technique options (a); the midline sinus is excised by asymmetric elliptical incision and a new flap is created by undercutting the fascia at the medial wound edge and (b), advancing it across the midline. By doing this, the natal cleft is flattened and the entire suture line is positioned lateral to the midline (c). (FCF - fascio-cutaneous flap, PSF - presacral fascia, $\mathrm{S}$ - sacrum).

cations such as infection, wound dehiscence, and seroma. Patients with acute inflammation and/or abscess formation were preoperatively treated with antibiotics and drainage, and then operated. All patients were operated under spinal or epidural anesthesia. A single dose of cefazoline $1 \mathrm{~g}$ was administered parenterally 30 minutes before skin incision. All patients were admitted to hospital the night before operation and treated with two enemas, particularly one at night and another three hours before operation. Patients were seen routinely for wound inspection in the surgical ward on postoperative days 3 , and 14. All procedures were approved by the local ethics committee and all subjects gave their informed consent to participate in the study. Three months after surgery, patients were invited to the hospital for follow-up. Time needed to return to work and period to complete the healing were recorded by us. Time needed to recuperate from pain when sitting on toilet and walking was obtained from all patients. The patients' responses were documented and analyzed. Patients were followed up for a mean period of $21.4 \pm 1.2$ months (range; 12 to 48). By the time of data analysis (January 2009), we were able to carry out a face-to-face interview with 51 of them in terms of recurrence assessment.

\section{Surgical technique}

This procedure consists of a bilaterally parallel elliptical excision, mobilization of full-thickness fascio-cutaneous flap from the median line of the wound, fixation of the base of flap to the sacrococcygeal fascia by methods of overlapping, and suture of its edge to the lateral side. At surgery, patients were placed in a prone jack-knife position, with the buttocks strapped apart with the use of adhesive tapes. Firstly, we shaved the sacrococcygeal area and we cleaned this area with povidone-iodine. The extent of the sinus was assessed by a stylet and a small amount of methylene blue was injected into the sinus to outline the cavity. The area to be excised $(A B C D)$ was mapped on the skin as in pattern shown in Figure Ia, including the orifices of the sinus tracts. Secondly, the excision was carried out downward to the presacral fascia and laterally until normal fat tissue was reached. Thirdly, a fascio-cutaneous flap was harvested from the gluteal muscle on $\mathrm{ABC}$ line (Figs 1a and 1b). The flap's diameter was equal to that of excised area $(A B C D)$ (Figs $1 b$ and $2 b$ ). Then, we removed the adhesive bands and we advanced this flap from the medial line laterally beyond the ADC line (Fig. 1c). After this maneuver, we sutured this flap with 0 polyglicolic acid onto the presacral fascia by us- 
ing the method of overlapping (Fig. 2). We performed a relaxation incision into subcutaneous tissue when we felt tension. Then, the subcutaneous tissue was sutured with polyglycolic acid suture. Finally, we closed the skin with the use of interrupted polypropylene suture (Fig. 1c). We put the drain in 5 patients treated with a flap of 7 to $10 \mathrm{~cm}$ in width.

All patients were mobilized in the fist postoperative hour. Independent waking up and going to the bed, sitting, walking and urination were allowed for patients. On postoperative days 3 , and 14 , the patients were seen routinely in the surgical ward for wound inspection and removal of sutures. Patients were recommended to return if they had any complaints thereafter. The skin sutures were removed on postoperative day 14 .

The cosmetic satisfaction was assessed according to patient's own description. Dissatisfaction is defined as an expressed cosmetic appearance of the scar in the surgical region.

\section{Results}

The mean age of the patients was $25.3 \pm 4.0$ years (range, 17 to 41). Five patients underwent surgery for recurrent PSD. The mean operation time was $32.0 \pm 5.0 \mathrm{~min}$. (range, 25 to 37 ). The mean length of defects after operations was $14.4 \pm 1.2 \mathrm{~cm}$ (range 8 to 20 ), and its mean width was $7.2 \pm 1.5 \mathrm{~cm}$ (range 4 to 10). Suction drains were removed when the drainage amount decreased below $15 \mathrm{ml}$ per day.

Complications, such as infection, wound dehiscence and seroma were detected in $2(3.5 \%), 2(3.5 \%)$ and $3(5.7 \%)$ patients, respectively. We did not observe suture reaction and flap necrosis in the patients. The mean durations of hospitalization, absence from work, and complete healing were $2.4 \pm 3.2$ (range, 2 to 3 ), 12.6 \pm 3.1 (range, 7 to 18 ) and $10.4 \pm 3$ (range, 5 to 17) days, respectively.

When we evaluate the time needed to recuperate from pain when sitting on toilet and walking, the results were $7.4 \pm 3.2$ days (range 4 to 10 ) and 6.5 \pm 2.7 days (range 4 to 9), respectively.

Average follow up period was $21.4 \pm 1.2$ months (range; 12 to 48$)$. PSD recurred in one $(1.7 \%)$ of the patients. Seroma was detected by clinical examination and in each case, it resolved spontaneously. We detected mild degree wound dehiscence in patients with wound infection $(n=2,3.5 \%)$. In these patients, the dehisced wound recovered by formation of granulation tissue after efficient wound care.

When we assessed the cosmetic satisfaction, no patient declared dissatisfaction with the cosmetic appearance of the scars.

\section{Discussion}

PSD is a frequent disease in young people. It has a high rate of morbidity causing long-term loss of working ability. It is a chronic inflammatory process of the skin caused by hair, keratin plugs, and debris (12). It is known that PSD incidence varies across countries and races. This may be because of different races. The incidence is also affected by factors such as driving, personal hygiene, and obesity (13). There are two conflicting theories of pathogenesis, particularly congenital and acquired. It is very important to understand the difference between these two possibilities because each requires different principles of treatment (14). Recently, the theory of acquired PSD has been widely accepted. Available evidence strongly supports the view that common adult PSD is an acquired condition resulting from hair insertion process $(6,14)$.

A study by Karydakis has reported that hair insertion at the depth of the natal cleft, the raphe, is the real cause of PSD (6). The author described three main factors contributing to the hair insertion process, particularly (a) the invading hair ( $\mathrm{H}$ factors), (b) the force of friction caused by the depth and narrowness of the natal cleft together with friction movements between its sides causing the hair to insert (F factors), and (c) the vulnerability of the raphe (V factors) (14). He claimed that these factors mentioned above should be decreased and/or neutralized to decrease the vulnerability of the raphe by replacing it with healthy skin while the midline wound scar should not be at the depth of the natal cleft.

New surgical techniques, with lower recurrence rates, aim to reduce the depth of natal cleft. There are several full-thickness flap techniques including excision and Z-plasty, W-plasty, V-Y advancement flap and transposition flaps such as gluteal myocutaneous and Limberg flaps (15-17). Several studies reported the recurrence rates of these techniques to vary from 0 to $5 \%$ (18, 21). The most frequently reported disadvantages include a wide angle of the extended incision in these flap techniques bringing about tensed sutures lines, and less desirable aesthetic outcomes $(5,18-21)$.

Our technique is developed on the basis of local defenses against hair insertion. It could be further maximized by decreasing or neutralizing the force factor as well. In this technique, the harvested flap could be fixed by suturing it to the lateral edge of the sacrococcygeal fascia, thus producing optimal flattening of the natal cleft. Additionally, we easily removed the vulnerable raphe. The maneuver provided us with a bilaterally parallel elliptical fascio-cutaneous advancement flap with the wound located as far as midline. In addition, by creating a force field in the vertical direction, the bilaterally parallel elliptical fascio-cutaneous advancement flap technique brings about a resistance (with a 90-degree angle) to force factors working in the longitudinal direction. Thus, our technique helps to decrease the friction movements via reducing the depth and narrowness of natal cleft. In this way, the hair insertion could be minimized and our main objective could be met, particularly to correct the two main etiologic factors in hair insertion as reported by Karydakis.

Several flap techniques reported in literature, have significantly reduced the patient absence from work and thus in the recent years, these techniques have gradually been attracting the attention of patients with PSD (5, 21-22). In our study, the patients were discharged after the surgery in 2.4 days on average and returned to work in 12.6 days on average with minimal incidence of morbidity. We believe that our technique could be advocated as an adjunct to primary closure to keep the incision away from the natal cleft and to flatten the natal cleft with reduced buttock friction. In addition, the excision with primary closure offered a potential to complete the healing for most of the patients within two weeks. 
In the conventional primary closure method, two main complications are important, particularly (1) high recurrence rate, and (2) wound dehiscence (23-24). In our study, there were only two minimal lower edge wound dehiscences with infection. These two cases were cured by second intention without requiring additional procedures. In our patients, there was only one case of recurrence $(1.7 \%)$. The patient had previous infections and the recurrence developed in six months after the surgery. The patient underwent surgery with the same technique and we did not observe recurrence during the postoperative follow-up period. The reported mean hospitalization durations for Limberg flap and primary closure techniques are 3.4 and 4.6 day, respectively (23). However, our patients' hospitalization time was considerably lower (2.4 days).

Our technique has two main differences from Karydakis's method, particularly (a) minimal drainage necessary based on fullthickness fascio-cutaneous flap usage, and (b) potential to be used in widely affected region based on the usage of advancement flap.

\section{Conclusion}

Our novel technique provided minimum postoperative morbidity, short hospital stay and reduced absence from work. The technique also has a satisfying aesthetic outcome and decreased recurrence rate. Moreover, the flap could be prepared easily. In the light of our results, we suggest that our novel surgical technique seems to be a reasonable method in the treatment of PSD.

\section{References}

1. Hull TL, Wu J. Pilonidal disease. Surg Clin North Am 2002; 82 (6): 1169-1185.

2. McCallum I, King PM, Bruce J. Healing by primary versus secondary intention after surgical treatment for pilonidal sinus. Cochrane Database Syst Rev 2007; 17 (4): CD006213.

3. Akinci OF, Coskun A, Uzunköy A. Simple and effective surgical treatment of pilonidal sinus. Dis Colon Rectum 2000; 43 (5): 701-707.

4. Lahooti M, Taheri PA, Nezami BG, Assa S. Sacrococcygeal Pilonidal Sinus Treated by a New Fascio-Cutaneous Flap. Dis Colon Rectum 2008; 51 (5): 588-592.

5. Eryilmaz R, Sahin M, Alimoglu O, Dasiran F. Surgical treatment of sacrococcygeal pilonidal sinus with the Limberg transposition flap. Surgery 2003; 134 (5): 745-749.

6. Karydakis GE. New approach to the problem of pilonidal sinus. Lancet 1973; 22 (2): 1414-1415.
7. Bascom J. Pilonidal disease: origin from follicles of hairs and results of follicle removal as treatment. Surgery 1980; 87 (5): 567-572.

8. Unalp HR, Derici H, Kamer $\mathbf{E}$ et al. Lower recurrence rate for Limberg vs. V-Y flap for pilonidal sinus. Dis Colon Rectum 2007; 50 (9): 1436-444.

9. Mentes O, Bagci M, Bilgin T et al. Limberg flap procedure for pilonidal sinus disease: results of 353 patients. Langenbecks Arch Surg 2008; 393 (2): 185-189.

10. Berkem H, Topaloglu S, Ozel H et al. V-Y advancement flap closures for complicated pilonidal sinus disease. Int J Colorectal Dis 2005; 20 (4): 343-348.

11. Tezel E. A new classification according to navicular area concept for sacrococcygeal pilonidal disease 2007; 9 (9): 575-576.

12. Sondeneaa K, Pollard ML. Histology of chronic pilonidal sinus. APMIS 1995; 103 (4): 267-272.

13. Buie LA. Jeep disease. South Med J 1994; 37 (2): 103-109.

14. Karydakis GE. Easy and successful treatment of pilonidal sinus after explanation of its causative process. Aust N Z J Surg 1992; 62 (5): 385-389.

15. Roth RF, Moorman WL. Treatment of pilonidal sinus and cyst by conservative excision and W-plasty closure. Plast Reconstr Surg 1977; 60 (3): 412-415.

16. Schoeller T, Wechselberger G, Otto A, Papp C. Definite surgical treatment of complicated recurrent pilonidal disease with a modified fasciocutaneous V-Y advancement flap. Surgery 1997; 121 (3): 258-263.

17. Manterola C, Barroso M, Araya JC, Fonseca L. Pilonidal disease: 25 cases treated by the Dufourmentel technique. Dis Colon Rectum 1991; 34 (8): 649-652.

18. Urhan MK, Kücükel F, Topgul $K$ et al. Rhomboid excision and Limberg flap for managing pilonidal sinus: results of 102 cases. Dis Colon Rectum 2002; 45 (5): 656-659.

19. Erdem E, Sungurtekin U, Nessar M. Are postoperative drains necessary with the Limberg flap for treatment of pilonidal sinus? Dis Colon Rectum 1998; 41 (11): 1427-1431.

20. Bozkurt MK, Tezel E. Management of pilonidal sinus with the Limberg flap. Dis Colon Rectum 1998; 41 (6): 775-777.

21. Mansoory A, Dickson D. Z-plasty for treatment of disease of the pilonidal sinus. Surg Gynecol Obstet 1982; 155 (3): 409-411.

22. Allen-Mersh TG. Pilonidal sinus: finding the right track for treatment. Br J Surg 1990; 77 (2): 123-132.

23. Ertan T, Koc M, Gocmen $\mathbf{E}$ et al. Does technique alter quality of life after pilonidal sinus surgery? Am J Surg 2005; 190 (3): 388-392.

24. Mentes O, Bagei M, Bilgin T et al. Management of pilonidal sinus disease with oblique excision and primary closure: results of 493 patients. Dis Colon Rectum 2006; 49 (1): 104-108. 